\title{
Sequencing the salmon genome: A deliberative public engagement
}

\author{
KIERAN O’DOHERTY, MICHAEL BURGESS, DAVID M. SECKO ${ }^{1}$
}

\begin{abstract}
Salmon genomics is an emerging field that represents a convergence between socially important scientific innovation and a politically volatile topic of significant interest to the public. These factors provide a strong rationale for public input. This report describes such input from a public engagement event based on the principles of deliberative democracy. The event involved a random, demographically stratified sample of 25 British Columbians (Canada). While some participants opposed sequencing the salmon genome on principle, on the whole participants responded favourably, citing the value of increased knowledge of human impacts on salmon, potentially slowing or reversing this impact, and assisting sustainable management of salmon resources. Participants discussed many of the potential implications of the sequencing project and formulated recommendations pertaining to regulations, international treaties, public education and engagement. Overall, the project illustrates that the specific design and implementation of this event is capable of producing sound policy advice from the general public on genomic-related issues.
\end{abstract}

\section{Introduction}

Current efforts towards funding an international project to sequence the Atlantic salmon (Salmo salar) genome present a critical point for advancing knowledge about salmonids (salmon, trout, and charr). ${ }^{2,3}$ Salmonids are one of the most studied fish on the planet. Their scientific interest in terms of evolution, genome duplication and genetics (for example) has produced more than 20,000 reports in the past 20 years. ${ }^{4}$ Scientific interest has been complemented with an interest in the environmental, economic and social importance of salmonids, including their popularity as a food choice, their traditional importance for various indigenous populations, their being sought by sports fishermen and their being part of a healthy ecosystem. Salmonids are also a topic of passionate debate related to the decline of salmon runs in the Pacific Northwest, the environmental effects of salmon aquaculture, best practices for commercial and subsistence fishing, and the production of transgenic salmon for human consumption. ${ }^{5,6,7,8}$ The sequencing of the Atlantic salmon genome thus presents an opportunity to study the values underlying public opinion on the subject, as well as specific interests in salmon aquaculture and conservation.

Arguably two of the most controversial issues relating to salmon are the practice of salmon aquaculture and the production of transgenic salmon. These two issues converge with the production of AquAdvantage salmon, a patented transgenic variety of Atlantic salmon with a maturation rate of four to six times that of the unmodified variety. ${ }^{9}$ The increased maturation rate is due to the insertion of a growth hormone gene from a Pacific Chinook salmon and a promoter sequence derived from the ocean pout. Although the issue has already been under consideration for many years, the FDA recently announced further public consultations on whether - if approved for human consumption - transgenic salmon such as AquAdvantage salmon will need to 
be specifically labelled. ${ }^{10}$ Transgenic salmon is only one particular genetic technology involving salmon (note, for example, DNA barcoding ${ }^{11}$ ), and indeed such work does not require the full sequencing of the Atlantic salmon genome. More interesting from a social scientific perspective is the geneticisation of yet another domain of life with reference to salmon. ${ }^{12}$ As argued by Holloway and Morris, genetics and genomics represent a particular way of knowing life. ${ }^{13}$ Specifically in the context of the practices of characterising livestock agriculture, they describe the shift that has taken place, from visual appraisal of animals and maintaining performance records to genetic and genomic techniques to assess particular aspects of animals' genotypes. This, they argue, is associated with a change towards distinctive ways of understanding animals, engaging with them, and representing them in society. Although there is contention as to whether genetic and genomic techniques should be understood as fundamentally different from more traditional selective breeding practices, they are arguably part of the geneticisation evident in many aspects of society.

These factors provide a strong rationale for public input on these issues. This paper describes the results of a public engagement event based on the principles of deliberative democracy on the topic of salmon genomics (entitled "Sequencing the Salmon Genome: A Deliberative Public Engagement" or "Salmon Genetalk" for short). Deliberative democracy is an approach to developing policy advice that emphasizes the inclusion of informed citizens and a two-way interaction between decision-makers and the public (described further in section 2.1 below).

There are several elements to the rationale behind conducting such a public deliberation. First, issues surrounding salmon are prominent in the public sphere (e.g., aquaculture, conservation, aboriginal rights), making it important to understand the range of perspectives to be taken into account in formulating policy pertaining to these issues. Such informed policy is more likely to be publicly acceptable and therefore politically viable. A deliberative public engagement provides a mechanism for such input from the public by producing group decisions from an informed 'minipublic'. ${ }^{14}$

Second, with little awareness of salmon genomics among the general public, ${ }^{15,16}$ an informed public engagement may anticipate future concerns related to the subject. Layering engagements on the issue of sequencing, and conducting deliberations among diverse groups, may provide valuable insights and a first step to resolutions.

Third, such research is an important opportunity to understand the social processes underlying deliberation on biotechnologies intended for controversial uses and politicised domains. In this context, the salmon deliberation represents a contrast to other topics and in particular the BC Biobank Deliberation. ${ }^{17}$ This event was hosted by the authors in 2007 and focused on biobanking, a topic with a similarly low public profile, but a much less controversial starting point compared to salmon (a highly politicised topic in British Columbia).

Taken together, these elements encompass our rationale in seeking policy advice from citizens of British Columbia (BC), Canada, on the social implications of the 
sequencing of the salmon genome. While effective utilization of deliberative public advice remains challenging ${ }^{18}$, this public engagement provides further evidence for the hypothesis that it is possible to have meaningful informed deliberation with randomly selected members of the public on complex biotechnological issues.

\section{Methods}

\subsection{Deliberative Democracy}

Public deliberation has a long and celebrated history from the city states of ancient Greece to the town hall meetings of colonial New England. ${ }^{19}$ Within this history, deliberative democracy has emerged as a subfield in political theory, including a practical orientation assessing the application of deliberative theory to the design, implementation, and evaluation of deliberative forums. As such, deliberative democracy has also become a valuable framework for the staging of public consultations that inform science and technology governance. ${ }^{20}$

The desirability of involving lay persons in policy and decision making in science, medicine and technology has been discussed in a significant amount of literature. ${ }^{21,22}$ Input from lay publics is needed to develop just and sustainable collective decisions about new science and technology, taking into account multiple value systems and considering potential impacts on different individuals and communities. Such considerations are promoted in a number of ways, ${ }^{23,24,25,26}$ such as stimulating citizenship and addressing 'democratic deficits' in policy development through deliberative democracy exercises. ${ }^{27,28,29}$ In this context, deliberative democracy is a process of learning, debate and collective decision-making on a topic. Processes underlying deliberation focus on respectful engagement between participants, positions taken by participants being justified and challenged by others, and conclusions that represent the deliberating groups' efforts to arrive at decisions without compromise that is unacceptable to some participants. The fundamental objective is a process for citizen participation that enables discourse while avoiding manipulation. ${ }^{30}$ Features that differentiate deliberation from other forms of conversation include: (i) debate or discussion that is aimed at producing reasonable, well-informed opinions, (ii) participants being willing to revise their position in response to such discussion and new information, and (iii) an overriding interest in the legitimacy of the outcomes that emerge from such discussions. ${ }^{31}$

Traditional social scientific methods for measuring public opinion include opinion surveys, interviews, and focus groups. While each has strengths and weaknesses, these methods have problems in common. For example, there is a gap between how people say they would respond and how they might actually respond in a given situation. In the context of policy options there can therefore be a gap between spontaneous expressions of opinion in a research study versus opinions expressed during considered and informed discussion of practical policy implications and a diverse range of relevant perspectives.

While no approach is perfect, the methodology described here attempts to move beyond simple aggregation of individual opinions, to informed group decisions about collective interests and the justification of opinions and policy. First, having people 
try to understand each others' reasons and give explanations for different positions provides an opportunity for their decisions to become collective rather than individual. That is, rather than methodologically constructing group opinion from aggregation of individual opinions taken out of context, group opinions are measured as they emerge from meaningful social interaction. Second, the tendency to produce superficial responses that may quickly change with additional information or challenge by others is reduced by engaging individuals in discussions with each other and providing multiple sources of information.

\subsection{Recruitment}

The goal of recruitment was to use demographic and screening questions to maximize the diversity of values, life experiences and communicational styles present in a stratified sample of 25 participants from British Columbia (BC) ${ }^{32}$ In the current study, a larger sample would have made deliberation more difficult to manage and, for all but the most important national issues, financially prohibitive. To achieve a sample that represented the diversity of BC, demographic stratifications were drawn from Statistics Canada and BC Statistics - Municipal Population Estimates. ${ }^{33}$ The sample was constructed by using random digit dialling to fill the required diversity across variables. The actual distribution of participants is shown in Table 1. Recruitment involved oversampling to 32 participants to account for attrition. 26 participants registered on the first day and 25 participants completed the event.

Table 1: Demographic stratification of participants

\begin{tabular}{|c|c|c|c|c|c|}
\hline \multicolumn{6}{|c|}{ Participant Demographics } \\
\hline \multirow[t]{2}{*}{ Gender } & Female & 12 & \multirow[t]{11}{*}{ Occupation } & Not working & 6 \\
\hline & Male & 13 & & Aquaculture & 3 \\
\hline \multirow[t]{2}{*}{ Region } & Greater Vancouver & 14 & & Construction & 2 \\
\hline & Rural BC & 11 & & Manufacturing & 1 \\
\hline \multirow[t]{11}{*}{ Age } & $<20$ & 1 & & Wholesale trade & 1 \\
\hline & $20-24$ & 0 & & Retail trade & 0 \\
\hline & $25-29$ & 0 & & Finance and real estate & 1 \\
\hline & $30-34$ & 0 & & Health care and Social services & 2 \\
\hline & $35-39$ & 2 & & Educational services & 3 \\
\hline & $40-44$ & 5 & & Business services & 0 \\
\hline & $45-49$ & 5 & & Other & 6 \\
\hline & $50-54$ & 2 & \multirow[t]{6}{*}{ Religion } & Catholic & 2 \\
\hline & $55-59$ & 2 & & Protestant & 3 \\
\hline & $60-64$ & 4 & & Christian, other & 5 \\
\hline & $>65$ & 4 & & Sikh & 1 \\
\hline \multirow[t]{4}{*}{ Ethnicity } & Chinese & 2 & & Other religious affiliation & 2 \\
\hline & South Asian & 2 & & No religion & 12 \\
\hline & Aboriginal & 3 & & & \\
\hline & Non minority & 18 & & & \\
\hline
\end{tabular}




\subsection{Information Materials}

There has been increasing criticism of the 'deficit model' of public understanding of science. ${ }^{34}$ This criticism challenges the belief that public resistance to biotechnologies is the result of insufficient information or understanding. When the deficit model is used in the context of public engagement activities, it can lead to approaches that simply desire to inform the public in anticipation that this will allay fears, without seeing a need to provide any real opportunity to influence policy. We acknowledge that accessible information is needed to deliberate meaningfully on complex biotechnologies. Significantly, however, providing information is, in the context of this project, not seen as an end in itself, but rather as a step in creating an environment in which meaningful deliberation takes place.

Providing appropriate and accessible materials, both in terms of content and amount, requires taking into account that materials should be:

- Unbiased (this is especially important for controversial topics like salmon);

- Representative of the diversity of views available on the topic, both in the public sphere and expert literature;

- Accessible and varied to take into account differences in learning styles.

To achieve these aims, participants were provided with:

1. A booklet written specifically for the event describing salmon genomics, genome sequencing, and providing contextual information. The booklet was written in clear simple language, with a hard copy mailed to participants before the event;

2. An annotated bibliography of relevant papers from the peer-reviewed literature and government documents;

3. A collection of media articles on the topic;

4. Presentations from five speakers representing expert knowledge on technical issues and stakeholder views. Speakers were only present on the first day and did not participate in deliberations. This was to avoid mixing lay and expert voices, which has been shown to marginalise the views of non-experts; ${ }^{35,36}$

5. A model used as an educational aid to illustrate the contextual landscape surrounding salmon. The model illustrated the different groups who have an interest in salmon (First Nations, commercial and recreational fishing, aquaculture, etc.), the importance of salmon as a cultural symbol, and its ecological importance;

6. A website to which participants were directed during the recruitment process, with a private area accessible only to confirmed participants. The private site included information and a forum for participants to interact with each other and the research team. One-on-one instructions on how to use the website were provided;

7. A dinner providing a comparison of farm-raised Atlantic, wild-caught Spring, and Coho salmon, accompanied by an explanation from the chef. 


\subsection{Event Design}

The deliberative event was staged over two non-contiguous weekends in November, 2008. The first day was geared to informing and orienting participants with speaker presentations; days two, three, and four were directed solely towards providing participants with a forum to discuss their views on the subject among themselves. From the second day, participants were split into three small groups in which they conducted their deliberations. The members of each small group did not change over the course of the event. A professional moderator facilitated the large group discussions, while three members of the research team facilitated the small group discussions (see below, 'Participant Tasks', for a description of the organisation of discussions). Between the two weekends, participants were encouraged to explore issues with each other (via the private website) and with friends and family.

The first weekend was intended to develop communication among participants to support respectful articulation and mutual understanding of differences of opinion. Small group discussions were intended to facilitate comfort and maximize participants' ability to express themselves and engage with each other. Large group discussions worked to establish common understanding, whether of technical or stakeholder perspectives, or that of the participants. A final post-event interview was conducted with each participant via telephone between six and eight weeks later (depending on availability of individual participants).

\subsection{Participant Tasks}

Deliberative democracy draws on a substantial body of theory and practice. ${ }^{37}$ The objectives of deliberation include participants being respectful of each other as equals, positions taken by participants being justified and challenged, and conclusions being a reflection of the deliberating groups' efforts to find common ground. ${ }^{38,39,40}$ Thus, in contrast to ordinary conversation or even structured debate, the particular characteristics of successful deliberation require that participants attempt to work towards collective resolutions to problems. Especially on complex issues, deliberation needs to be well informed about technical aspects without undermining the expectation that participants can redirect assumptions made by experts or stakeholders (see Section 2.3).

Several features were intended to provide important support for deliberation. For instance, one of the purposes of providing background materials and responses to technical questions was to enhance participants' confidence and knowledge. Further, recruiting for diversity of life experience, and the facilitation of discussions, was aimed at including and encouraging diverse reasoning styles in the group.

A particular challenge in designing public engagement is identifying the way in which tasks should be presented or questions posed to participants. ${ }^{41}$ Some engagements have been criticised for framing the questions to be deliberated in such a way that participants were not able to explore a full range of views and options pertaining to broader topics. We worked to address this problem by framing the two primary tasks (questions) for participants very broadly. The first task was for participants to explore their hopes and concerns for the sequencing of the salmon genome (day 2). The second was to deliberate on sequencing the salmon genome and work towards 
collective opinions (days 3 and 4); if there was in principle support, participants were asked to express the conditions under which they felt salmon sequencing should be conducted and, if they felt it should not proceed, document their reasoning. This second task was undertaken with the aim of achieving consensus among the participants in each small group (though this was not required). In other words, the first task was geared towards individuals expressing their individual opinions, with no further formal requirement for convergence. In contrast, the second task explicitly involved participants working towards producing collective positions.

The aim of the 'hopes and concerns' task was to accustom participants to listening to others and expressing their own views in a deliberative forum. This task was accomplished in small groups with reports to the large group. The hopes and concerns of each group was collated during the break between weekends and made available to participants for the second weekend.

The aim of the second task was to provide a focus to participants' deliberations and to lead to a coherent set of values and opinions that could be reported to policymakers. Nonetheless, the task was aimed to be broad enough that participants felt free to explore the range of issues they deemed relevant. The purpose of the consensus task was to move discussions beyond simply stating personal views, and to begin to arrive at collective decisions for meaningful policy input, requiring the negotiation of value trade-offs. In those instances where persistent disagreements emerged, facilitators did not seek consensus but encouraged participants to explore these differences and, if they could not be resolved, to document them clearly. This task was again initiated with discussions in small groups, followed by each group reporting their results to the large group as a whole.

Post-event, facilitators and notetakers from each small group produced reports based on the final participant presentations. These reports were distributed to participants who were asked either to ratify them or to note inaccuracies. In instances where individual participants responded based on personal opinion (rather than to point out an inaccuracy) these statements were noted for further analysis, but no amendments were made. Because there was insufficient time in the final discussion to reconcile the small group reports into a single collective statement from the large group, commonalities and differences between the small groups were examined analytically after the event and are documented below.

Participants were also asked to complete a 27 item survey that aimed to measure opinion on a variety of issues surrounding salmon and genomics. The survey was administered electronically via the NERD (Norm Evolution in Response to Dilemmas) platform ${ }^{42}$ before and after the deliberative engagement. In cases where participants did not have web access, a paper survey was administered. (Owing to the small sample size, the significance of providing a paper versus an electronic version of the survey could not be explored in this study.)

\subsection{Data Analysis}

All deliberations were audio recorded, transcribed, coded, and analyzed with the support of Atlas.ti software. Analysis of the survey was conducted with the aid of 
SPSS software. There were notetakers in each room for large and small group discussions. Several distinct analyses have been and are being conducted on the data, but space limitations allow us to report here on only two of these, one qualitative and one quantitative. Elsewhere ${ }^{43}$ we have argued that deliberative public engagements on complex issues can be understood as having two distinct kinds of output: analytical output, which is primarily a case of the researchers analysing data from the event according to areas of researcher interest, and deliberative output, which can be defined as a politically legitimate representation of the collective voice of the participants.

In the first part of the Results section of this paper (below) we focus on the deliberative outputs of the public engagement. That is, we provide a comprehensive summary of the collective statements agreed on by participants during deliberation and subsequently ratified by them during post-event phone interviews. These outputs are purposefully not elaborated by individual quotes, as these would not satisfy the criteria of representing ratified group opinion. Critically, the deliberative outputs also incorporate a description of those issues where no consensus was reached, with an explanation of the nature of the disagreement. In the second part of the results we provide a limited analysis of the survey responses with the aim of tracking significant shifts in opinion from pre to post event, as well as identifying issues that were notably resistant to opinion change in spite of participation in deliberation.

\section{Results}

\subsection{Deliberative Outputs}

For this event, the deliberative outputs constitute three reports based on the small group presentations (available upon request). The (self-appointed) names of the three groups were "Transposon", "Sockeye", and "Worriers (Warriors?)", referred to below as $\mathrm{T}, \mathrm{S}$, and $\mathrm{W}$.

As previously mentioned, the outcomes of the deliberation were the result of consideration of the following questions:

1) Should the salmon genome be sequenced or not?

2) If Yes, - How and under what conditions, if No, - Why not?

Even though the questions were centred on sequencing of the salmon genome, participants were encouraged to deliberate broadly about potential applications. Indeed, participants expressed difficulty conceptualising the sequencing of the salmon genome as a self-standing project, and felt it important to consider foreseeable applications associated with the sequencing.

Across each small group, there was overall support for the project of sequencing the salmon genome (conditions expressed by participants are discussed below). This support seemed to be based primarily on valuing the role that increased knowledge could have for understanding human impact on salmon, potentially slowing or even reversing this impact, and assisting sustainable management of salmon resources. There were individuals, however, who never wavered from their position of being 
opposed to the sequencing. Their positions were variously characterised as owing to "cultural values" and not "messing with mother nature." Ultimately, one small group (S) was strongly in support of the project with no one against; one was largely in favour (W) with only one member opposed; and one group was mixed (T).

Deliberation on the second question provides a richer record of responses to the social and ethical implications of salmon genomics. Discussions on these dimensions extended far beyond simple reactions to a proposed project to considered exploration of diverse public values relevant to the issue. The following is a summary of some of the main issues discussed by participants.

\section{Regulation}

Participants saw a strong need for updated and enforceable regulations, based on impartial and reasonable assessment of the risks associated with actual and potential applications of salmon genomics. Given the unknown long-term consequences of having a salmon genome and its uses, participants felt strongly about Canadian policymakers taking the lead internationally in assuring transparency and accountability to the public and to future generations.

A strong theme in discussions focused on the need for a unified regulatory body, preferably on the federal level, which would oversee salmon genomics research and applications. One small group (S) specifically called for a "watchdog" with the mandate of guarding the interests of the Canadian public. Another group (T) (independently) emphasised that such a body should operate from the default position that it is the duty of the researcher or developer to prove the safety of any future product. This group emphasised the need for any guidelines to be practically enforceable. Participants argued the need for a regulatory body based on observations that enthusiastic and well intentioned uses of technology in the past have led to serious problems (e.g., misuse of DDT).

\section{International treaties}

Participants recognised that Canada's waters are part of a world-wide system and thus saw a need for international treaties regarding the uses of genomic technologies in the context of salmon. Participants wanted Canadian policymakers to emphasise to the international community the need for precautionary, enforceable regulations, and to seek treaties to prevent risky and untested uses of salmon genomics. Participants expressed especially strong support for impartial oversight and regulations to be in place prior to extensive genetic modifications of salmon being developed.

\section{Public education and engagement}

Participants expressed a strong need for greater public awareness about many of the issues relating to the sequencing of the salmon genome. Suggestions included involving young children in activities such as walks to see salmon spawning or incorporating genomics issues into the curriculum of high school students. Finally, participants suggested fostering public awareness through government-sponsored media campaigns. An underlying factor in these recommendations was a call for adequate provision of information to the public to enable informed choice on matters such as food-labelling (see below) and to dispel irrational fears. 


\section{Labelling of salmon}

Although labelling does not pertain solely to salmon genomics, participants felt the issue was nonetheless relevant. In particular, participants felt very strongly that labelling for salmon should be mandatory, and that information regarding the farmed, wild, or GM (if this became available) nature of a salmon should be available to the consumer (S). Some participants also argued that, although the sequencing was clearly not a pre-requisite for the production of GM salmon, work on GM salmon would be supported by the knowledge produced from sequencing the salmon genome (T).

\section{Concerns}

Participants' calls for regulations, treaties, and public awareness were driven by various concerns they hoped could be addressed. Three concerns were dominant:

1. Sequencing the salmon genome is akin to "opening Pandora's Box", in the sense that it could lead to the increased likelihood of genetic modification of salmon on a commercial scale.

2. Environmental concerns were expressed with regards to increasing pressures on the sustainability of wild salmon based on current practices. Participants emphasised the importance of prioritising the environment/habitat of salmon, such that knowledge derived from sequencing the salmon genome should be used to help protect salmon habitats and restore balance to the ecosystem if possible.

3. Most participants recognized transgenic salmon as a separate issue from the sequencing of the salmon genome, and that the sequencing was not necessary for the production of transgenic fish. Nevertheless, the group's assessment was that, if anything, such research would facilitate work on transgenic salmon and increase the likelihood of its commercial production. Overall, most participants were strongly opposed to the commercial production of transgenic salmon.

\subsection{Survey Analysis}

All participants completed surveys to ascertain their views before and after the event, to support the assessment of the effects of information and deliberation (the group is too small to be treated as a sample for a survey of a population). The survey contained 27 items for which participants were asked to indicate their level of (dis)agreement ( 1 =strongly disagree; $2=$ disagree; $3=$ no opinion; $4=$ agree; $5=$ strongly agree; don't know/insufficient information). There are many directions from which these data can be approached and we provide here only a snapshot of some survey items where there was either (i) a significant shift or (ii) relatively strong consensus with little change from pre- to post-test.

We include this analysis to complement the qualitative analysis and to illustrate how opinions were tracked pre- and post-event, but it is outside the scope of this paper to explore the survey data further (a list of the survey questions is available at: http://salmongenetalk.com). It is important to note that the pre/post sample sizes (N) vary in the presented data due to the inclusion of a "don't know/insufficient information" response that is not included in the presented analysis. This "don't 
know" choice was included to distinguish between a neutral sentiment and a feeling of insufficient information. Such sample size variations are accounted for in the statistical analysis, but participant movement from the "don't know" choice to another option does complicate the interpretation of the data. Nevertheless, reported changes do support modest aggregate shifts in the group on indicated items as a result of deliberation.

A statistically significant change in opinion was observed on several topics related to salmon genomics, including new regulations, implications for First Nation peoples, labelling of salmon for consumption, the role of salmon genomics in making salmon farming more sustainable, and the importance of genetic diversity in wild salmon (Figure 1). After the deliberation more participants viewed the sequencing of the salmon genome as having a role in making salmon farming more sustainable (Q7; $z=$ $-2.021, p=0.043$ ), and more participants viewed the sequencing as a threat to First Nation peoples' knowledge and values (Q19; $z=-2.174, p=0.03)$. On a modest level, participants slightly shifted post-deliberation on the importance of maintaining genetic diversity in wild salmon (Q23; $z=-2.967, p=0.003)$, from a "disagree" to a "strongly disagree". Similarly participants showed a modest shift from "agree" to "strongly agree" on whether new regulations are required to address issues related to salmon genomics (Q6; $z=-2.145, p=0.032)$.

Figure 1: Significant changes in opinion
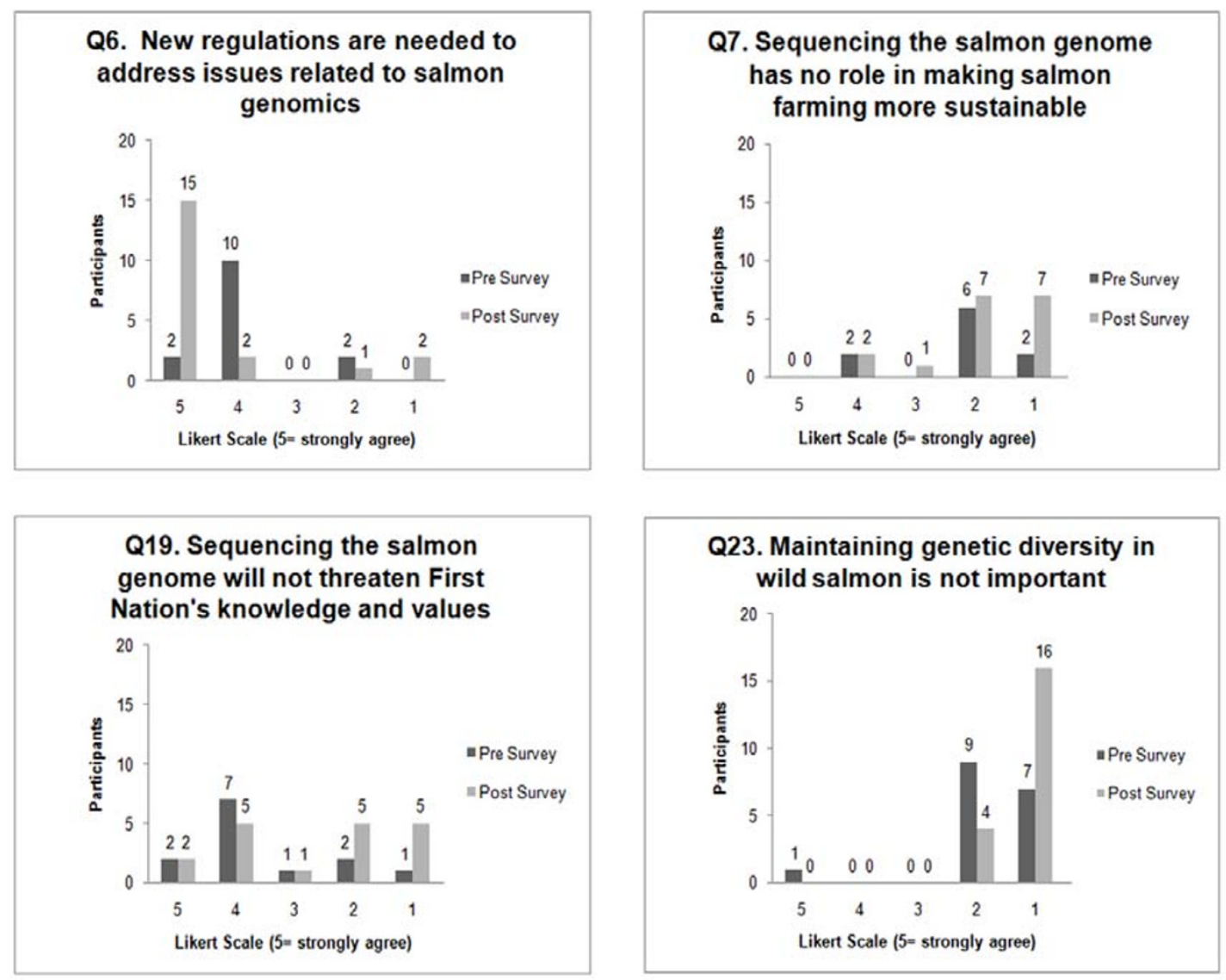
In contrast, certain items stood out as being associated with relatively uniform views across participants, and did not change as a result of the deliberation (Figure 2). In particular, before the deliberations 95 per cent of participants stated that protecting wild salmon should be a high priority in $\mathrm{BC}$, with this rising to 100 per cent postdeliberation (Q4). Most (90 per cent) participants either disagreed or strongly disagreed with the statement that it does not matter how salmon is produced as long as it is inexpensive both before and after the deliberation (Q20). Finally, labelling of salmon products, in spite of increased costs to the consumer, was important to participants, with 90 per cent responding to the statement with either 'Agree' or 'Strongly disagree' pre-event and 100 per cent post-event (Q21).

Figure 2: Unchanged opinions
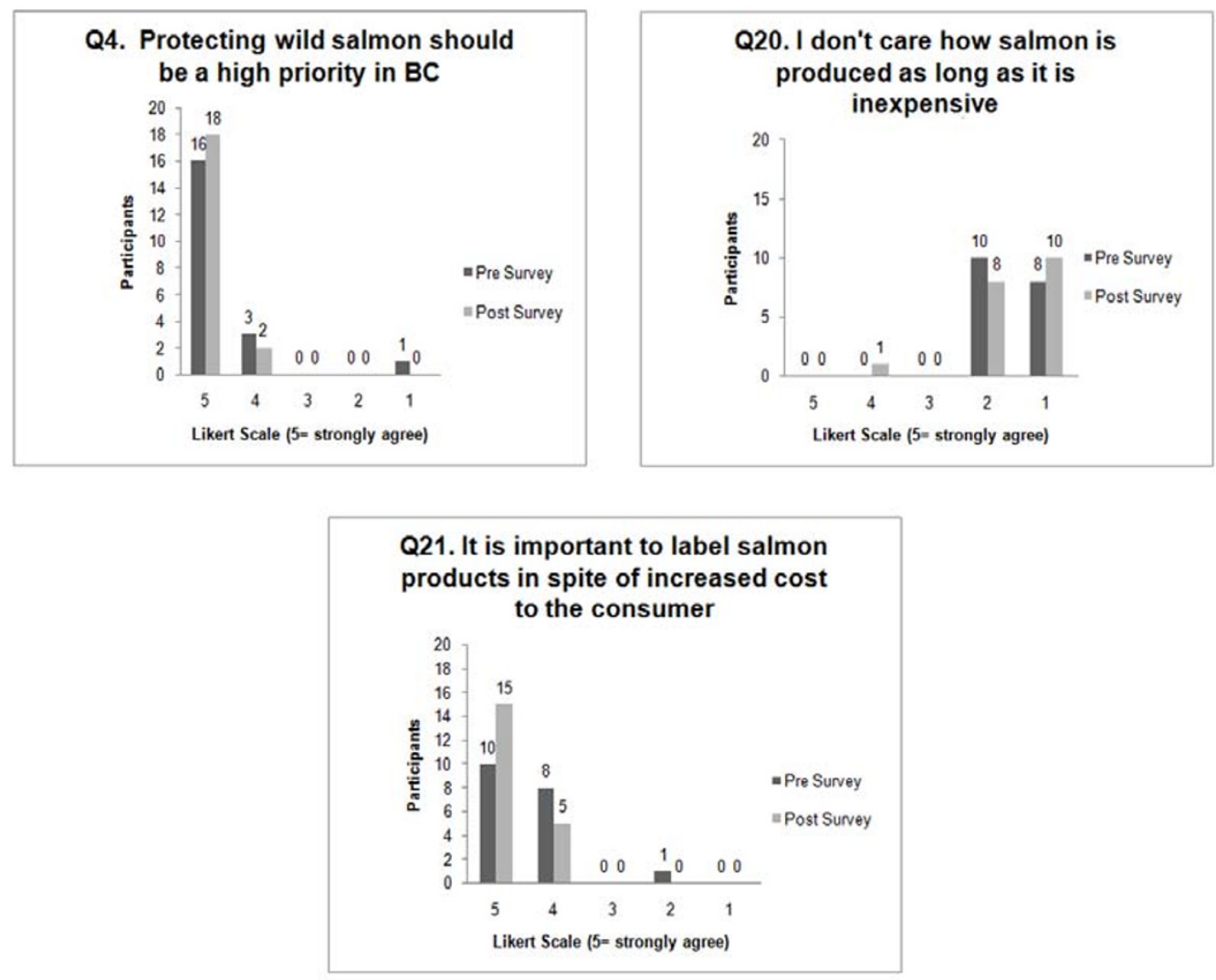

\section{Discussion and Conclusions}

Whether or not AquAdvantage salmon ever hit supermarket shelves, the geneticisation of salmon has had and will have an increasingly powerful effect on knowledge-practices and institutional relationships, similar to that described by Gibbs in the agricultural livestock context ${ }^{44}$. In this regard, this study on salmon provides an interesting counterpoint to social scientific studies of agricultural livestock. In contrast to sheep and cattle farming, salmon farming is itself still a controversial practice, at least in British Columbia. Morris and Holloway use Foucault's concept of biopower as a lens for examining the reconfigured relationships evident in cattle and sheep farming as a result of the availability and use of genetic and genomic technologies. ${ }^{45}$ They identify potential avenues of human and animal resistance to subjectification under this form of biopower. Our study suggests that while the production of 
transgenic salmon is resisted strongly by our participants, the genetic lens that salmon genomics offers for human understandings of salmon is generally not resisted. In fact, when genomic knowledge and techniques are promised to help ecological tasks, they are embraced. This is seen, for example, in how participants shifted post-deliberation to a belief that the salmon genome should have a role in making salmon farming more sustainable (Figure 1, Q7), which is perhaps drawn from the group maintaining a strong desire to protect wild salmon (Figure 2, Q4).

This is particularly interesting in the context of the Pacific Northwest and Western Canada, given the widespread controversy there surrounding salmon aquaculture. For those not familiar with this context, it is often surprising to note the frequent statements by restaurants in the region that they serve only 'wild salmon', and the vehement and sometimes vicious debates that can ensue there when willingness to eat farmed salmon is expressed. A consequence of this particular cultural view of salmon in $\mathrm{BC}$ is that, arguably, dominant segments of society currently resist what Twine calls a productivist paradigm in farm animal breeding ${ }^{46}$ (an emphasis on maximising efficiency in the derivation of animal products; in the context of this paper, see Figure 2, Q20). It is interesting to note that in the context of sheep and cattle Twine associates certain aspects of genomics discourse with a delegitimisation of the productivist paradigm; in contrast, the AquAdvantage salmon arguably embodies a poignant illustration of the productivist paradigm with its capacity for rapid maturation built into its very genetic make-up.

All of this is to say that budding salmon genomics technologies are emerging in a complex and contested social context, in which policymakers will have difficulty in developing regulations that will be accepted by diverse publics and interest groups. Inviting citizens to join policy-making processes through deliberative engagement introduces informed reflection from a diverse range of views, thereby enhancing policy legitimacy. Deliberations between culturally diverse participants have the potential to explore taken for granted assumptions of experts or majorities by challenging dominant perspectives and respectfully (as well as accurately) articulating other opinions, even in the face of disagreement. ${ }^{47}$ In this study, opposition to salmon genomics has been closely linked to concerns about particular applications (e.g. transgenic salmon) and how policies about those applications may reduce resistance to sequencing the salmon genome. Such deliberations are therefore able to respond to important, and potentially divisive, social issues and seek trade-offs and ways of living together that focus on collective well-being. Developing more inclusive approaches to policies related to the development of genomic and technological research on salmon not only fosters enhanced representation in policy making, but ultimately also produces more robust policy that is more likely to sustain broad public support.

In terms of participants' recommendations, there was general support for sequencing of the salmon genome. It should be noted, however, that a small number of participants maintained a position against the sequencing project based on concerns centring on (i) the environment and (ii) transgenic applications. Some of these concerns were shared by all participants, even those who supported the sequencing project. For instance, almost all participants rejected GM salmon, but only a few 
opposed the sequencing project based on arguments that it would facilitate transgenic applications. Moreover, concerns about the environment included recognition that salmon genomics might help alleviate some pressures on the sustainability of wild salmon stocks (which was also reflected in the post-event survey; Figure 1, Q7). Participants also deliberated over whether the resources going into genomic research might instead be used to combat pollution and other factors that have a negative impact on salmon. Participants' concerns about transgenics took into account that sequencing the salmon genome was not required for the creation of transgenic salmon and that such an application was not a goal of the scientific sequencing project. However, some participants were concerned that a full salmon genome would facilitate the development of transgenic fish. Thus, although there was strong support for the sequencing project, with only a few disagreeing, participants were strongly opposed to its use for the development and commercial production of transgenic salmon.

Another strong theme was the call for a federal regulatory body and international treaties. This is unsurprising given that some participants apparently entered the event with a sense that new regulations were required to address issues related to salmon genomics (Figure 1, Q6). According the post-survey results this sense increased during the event. We interpret these calls for collective action on a scale beyond the local level as indicative of participants' recognition of the need to formulate effective policy that transcends narrow partisan interests and requires international cooperation.

The participants' call for public education and further public engagement constituted another important output. Since the deliberative event made the importance of policy related to salmon genomics obvious, it is not surprising that one of the core recommendations called for more information for and engagement with the public. This should be interpreted with some caution since salmon genomics must clearly compete with other emerging technologies and a range of important policy issues in this regard. Nevertheless, the overall sentiment of the deliberating group clearly indicates that efforts towards public education on salmon genomics would likely be met with interest. The call for increased public education on salmon genomics from a public forum could be interpreted as ironic, given the above stated stance on the public deficit model of public understanding of science. ${ }^{48}$ While this irony is not lost on us (particularly in deliberants' calls for more information to "dispel irrational fears"), it is worth pointing out that the sentiment generally attached to these calls for more public education and information had less to do with a perception of the public as hopelessly ignorant and therefore unable to make important policy-related decisions, and more to do with feeling disempowered owing to being kept 'out of the loop' of advances in science and technology. Hence, it is important to highlight a common misreading of Wynne's criticism of the deficit model: the problem is not in the provision for supplying more information about science and technology to lay publics; rather, the problem lies in the assumption that lay publics are unable to make 'rational' decisions owing to the lack of such information. Despite some exceptions, the call for more information and education described in this study was interpreted by us as a desire to regain exposure to aspects of life from which participants felt increasingly alienated, owing, for instance, to dramatic advances in science and technology and the associated bureaucratisation of policy decision making. 
It is worth a brief note that pertains to deliberation on issues that did not lead to full consensus. Although facilitators worked towards consensus in group discussions, it was seen as important to identify those issues where consensus could not be reached and to achieve clear articulation of the conflicting positions. This is important to avoid 'shallow' consensus or some participants 'giving in' to majority views, as might have been the case had consensus been required. Such areas of disagreement should be of particular interest to policymakers. Consensus recommendations arising from this deliberation clearly represent a strong impetus for policy action in a particular direction. In contrast, points of disagreement should be seen as likely to characterise fundamental disagreements in values or interests among the population. It is precisely these areas, therefore, where policymakers should be most sensitive to public acceptability and the long-term social sustainability of policies.

In many respects, Salmon Genetalk was a replication of the BC Biobank Deliberation. ${ }^{49}$ In contrast to the BC Biobank Deliberation, however, Salmon Genetalk participants seemed to express a greater degree of cynicism and mistrust. The research design does not allow us to attribute this observation to any specific factor, but we speculate that an important contributing factor is the different purposes that are attributed to salmon genome sequencing versus biobanking. In particular, the ultimate application of biobanking is to support human health. Consideration of the ethical and social implications of biobanking focuses largely on the implementation of biobanks, not on whether there is inherent value in biobanking itself. ${ }^{50}$

In contrast, the applications derived from sequencing of the salmon genome focus largely on economic and environmental considerations, with a number of consequences. First, the presence of economic interests may be associated with scepticism and mistrust. Second, there is an apparent lack of congruence between the use of genomic technologies in conservation, as these may not address the causes of ecological damage. Third, competing economic interests in salmon (e.g., aquaculture versus fisheries) and perceived competing vested interests (e.g., conservation versus subsistence fishing) are associated with conflicting factual claims. These factors arguably provide a fertile ground for scepticism and mistrust, which became evident in the deliberations.

It would be a mistake, however, to imply that the Salmon Genetalk deliberation was characterised by cynicism and mistrust overall. On the contrary, participants expressed a strong sense of excitement and empowerment relating to their involvement. Many participants described a sense of optimism based on being able to participate in a deliberative event dedicated to the social and ethical implications of a complex genomic topic, in their role as citizens (rather than as experts).

In conclusion, we return to the rationale behind conducting this deliberative engagement.

1. Extending the design of the BC Biobank Deliberation to public deliberation on sequencing the salmon genome demonstrated that this public engagement format is suitable across both human and non-human genomics topics. We do 
not wish to claim that public deliberation is feasible or even appropriate for all controversial issues. Nor do we want to promote this particular public engagement design independent of context. However, we do feel confident in claiming that the design described in this study may be used as a best practice that can be adjusted to suit the contingencies of particular issues.

2. We have also demonstrated that this public engagement design can be used for topics that are known to be highly polarised and potentially divisive. In contrast to human tissue biobanking, there is a relatively high degree of public awareness in $\mathrm{BC}$ about issues related to salmon. This public visibility is associated with polarised views, politics, and conflicting factual claims. However, these factors did not inhibit deliberation. Participants were able to engage in respectful discussion, voicing their opinions and having them challenged, and worked together to attempt to find resolutions.

3. We have presented further supporting evidence for the hypothesis that it is possible to have meaningful informed deliberation with randomly selected members of the public on complex biotechnological issues.

4. Finally, with an increase in public engagement events on topics of science and technology, it is hoped that the appropriate consideration of citizen advice will lead to a genuine commitment to its meaningful inclusion in policy development.

\section{Acknowledgments}

The deliberative democracy research team is part of the Genome Canada and Genome BC funded project Building a GE3LS Architecture through The W. Maurice Young Centre for Applied Ethics at the University of British Columbia (Principal Investigators: M. Burgess and P. Danielson). Research team members providing essential theoretical and logistical support for the event: Dan Badulescu, Michael Burgess, Emma Cohen, Isaac Filate, Alice Hawkins, Holly Longstaff, Sacha Ludgate, Ania Mizgalewicz, Shauna Nep, Kieran O'Doherty, Alexis Paton, David Secko, and Elizabeth Wilcox. Particular thanks also to Rob Balshaw for assistance with statistical analysis.

The deliberative engagement was funded by Genome Canada, Genome BC, and the Consortium for Genomic Research on All Salmonids Project (cGRASP).

\footnotetext{
${ }^{1}$ W. Maurice Young Centre for Applied Ethics, University of British Columbia (K.O.); W. Maurice Young Centre for Applied Ethics and Department of Medical Genetics, University of British Columbia (M.B.); Department of Journalism, Concordia University (D.M.S.); correspondence to: dsecko@alcor.concordia.ca
} 
${ }^{2}$ N.L. Quinn et al. Assessing the feasibility of GS FLX Pyrosequencing for sequencing the Atlantic salmon genome. BMC Genomics 2008; 9: 404.

${ }^{3}$ W. S. Davidson, Y. Guiguen, C.E. Rexroad 3rd \& S.W. Omholt. Salmonid Genomic Sequencing Initiative: The case for sequencing the genomes of Atlantic salmon (Salmo salar) and rainbow trout (Oncorynchus mykiss). http://www.cgrasp.org/whitepaper050905.pdf

${ }^{4}$ Quinn et al, op. cit. note 2.

${ }^{5}$ R.T. Lackey. Pacific Northwest salmon: forecasting their status in 2100. Rev. Fisheries Sci 2003; 11: 35-88.

${ }^{6}$ R.T. Lackey. Economic Growth and Salmon Recovery: An Irreconcilable Conflict? Fisheries 2005; 30: 30-32.

${ }^{7}$ D. J. Noakes, L. Fang, K. W. Hipel, \& D.M. Kilgour. An examination of the salmon aquaculture conflict in British Columbia using the graph model for conflict resolution. Fisheries Manag Ecol 2003; 10: 123-137.

${ }^{8}$ P. Aerni. Risk, regulation and innovation: The case of aquaculture and transgenic fish. Aquatic Sci 2004; 66: 327-341.

${ }^{9}$ E. Marden et al. The Policy Context and Public Consultation: A Consideration of Transgenic Salmon. Int Assessment 2006; 6: 73-97.

${ }^{10}$ Food and Drug Administration (FDA). Background Document: Public Hearing on the Labeling of Food Made from the AquAdvantage Salmon:

http://www.fda.gov/Food/LabelingNutrition/FoodLabelingGuidanceRegulatoryInformation/TopicSpecificLabelingInformation/ucm222608.htm

${ }^{11}$ F. O. Costa \& G. R. Carvalho. The Barcode of Life Initiative: synopsis and prospective societal impacts of DNA barcoding of fish. Gen Soc Policy 2006; 2: 29-40.

${ }^{12}$ L. Gannett. What's in a cause? The pragmatic dimensions of genetic explanations. Biol \& Philosophy 199; 14: 349-74.

${ }^{13}$ L. Holloway \& C. Morris. Boosted bodies: Genetic techniques, domestic livestock bodies and complex representations of life. Geoforum 2008; 39: 1709-1720.

${ }^{14}$ R.E. Goodin \& J.S. Dryzek. Deliberative Impacts: The Macro-Political Uptake of Mini-Publics. Politics Soc 2006; 34: 219-244.

${ }^{15}$ M.M. Burgess \& J. Tansey. Complexity of public interest in ethical analysis of genomics: Ethical reflections on salmon genomics/aquaculture. Integrated Assessment 2006; 6: 37-57.

${ }^{16}$ J. Tansey \& M.M. Burgess. The meanings of genomics: a focus group study of "interested" and lay classifications of salmon genomics. Pub Understand Sci 2008; 17: 473-484.

${ }^{17}$ M.M. Burgess, K.C. O’Doherty \& D.M. Secko. Biobanking in BC: Enhancing discussions of the future of personalized medicine through deliberative public engagement. Personalized Med 2008; 5: 285-296.

${ }^{18} \mathrm{~J}$. Abelson, et al. Deliberations about deliberative methods: Issues in the design and evaluation of public participation processes, Soc Sci Med 2003; 57: 239-251.

${ }^{19}$ M. X. Delli Carpini, F. Lomax Cook \& L. R. Jacobs. Public deliberation, discursive participation, and citizen engagement: A review of the empirical literature. Ann Rev Pol Sci 2004; 7: 315-344.

${ }^{20}$ P. W. Hamlett. Technology Theory and Deliberative Democracy. Sci Tech Human Values 2003; 28 : $112-140$

${ }^{21}$ H. M. Collins \&. R. J. Evans. The Third Wave of Science Studies: Studies of Expertise and Experience. Soc Studies Sci 2002; 32: 235-96.

${ }^{22}$ Jasanoff, S. Technologies of Humility: Citizen Participation in Governing Science. Minerva 2003; 41: 223-244.

${ }^{23}$ E. F. Einsiedel, Assessing a Controversial Medical Technology: Canadian Public Consultations on Xenotransplantation. Pub Understand Sci 2002; 11: 315-331.

${ }^{24}$ B. Myskja. Lay Expertise: Why Involve the Public in Biobank Governance? Genomics, Society and Policy 2007; 3: 1-16.

${ }^{25}$ G. Rowe \& L. Frewer, L. A Typology of Public Engagement Mechanisms. Sci Tech Human Values 2005; 30(2): 251-290.

${ }^{26}$ D.M. Secko, M.M. Burgess \& K.C. O’Doherty. Perspective on engaging the public in the ethics of emerging biotechnologies: From salmon to biobanks to neuroethics. Account Res 2008; 15: 283-302.

${ }^{27}$ M. M. Burgess \& J. Tansey, 2008. Democratic Deficit and the Politics of "Informed and Inclusive" Consultation. In Hindsight and Foresight on Emerging Technologies (E. Einsiedel \& R. Parker, eds.). Vancouver, BC: UBC Press, pp. 275-288. 
${ }^{28}$ J. Fishkin \& P. Laslett. 2003. Debating Deliberative Democracy. Malden, MA: Blackwell.

${ }^{29}$ J. Gastil \& P. Levine. 2005. The Deliberative Democracy Handbook: Strategies for Effective Civic Engagement in the Twenty-First Century. San Francisco, CA. Jossey-Bass.

30 J. Dryzek. 1990. Discursive Democracy: Politics, Policy and Political Science. Cambridge. Cambridge University Press.

${ }^{31}$ S. Chambers. Deliberative Democratic Theory. Ann Rev Political Sci 2003; 6: 307-326.

${ }^{32} \mathrm{H}$. Longstaff \& M.M. Burgess. Recruiting for representation in public deliberation on the ethics of biobanks, Pub Understand Sci 2010; 19: 212-224.

${ }^{33}$ BC Stats. Population Estimates. http://www.bcstats.gov.bc.ca/data/pop/pop/estspop.asp\#totpop

${ }^{34}$ B. Wynne. Public uptake of science: a case for institutional reflexivity. Pub Understand Sci 1993; 2 : 321-337.

${ }^{35}$ A. Kerr, S. Cunningham-Burley \& R. Tutton. Shifting Subject Positions: Experts and Lay People in Public Dialogue. Social Studies Sci 2007; 37: 385-411.

${ }^{36}$ K.C. O'Doherty \& H.J. Davidson. Subject positioning and deliberative democracy: Understanding social processes underlying deliberation. J Theor Soc Behav, 2010; 40: 224-245.

${ }^{37}$ see, for example, Gastil \& Levine, op. cit. note 29.

${ }^{38}$ Chambers, op. cit. 29; Dryzek, op. cit. 28; Gastil \& Levine, op. cit. note 29.

${ }^{39}$ P. W. Hamlett. Technology theory and deliberative democracy. Sci Tech. Human Values 2003; 28: 112 .

${ }^{40}$ J.K. Dryzek. 2000. Deliberative Democracy and Beyond: Liberals, Critics, Contestation. Oxford, UK. Oxford University Press.

${ }^{41}$ K.C. O’Doherty \& A. Hawkins, Making public engagement effective in policy development on human tissue biobanking. Pub Health Genomics, 2010;13: 197-206.

${ }^{42}$ P. Danielson. 2007. A Platform for Experiments in the Ethics of Science and Technology. In Sapporo Proceedings. K. Ishihara \& S. Majima, eds. Centre for Applied Ethics and Philosophy (CAEP) Hokkaido University, Sapporo, pp. 30-56.

${ }^{43}$ K.C. O'Doherty \& M.M. Burgess. Engaging the public on biobanks: Outcomes of the BC Biobank Deliberation. Pub Health Genomics 2009; 12: 203-215.

${ }^{44} \mathrm{D}$. Gibbs et al. Genetic techniques for livestock breeding: Restructuring institutional relationships in agriculture. Geoforum 2009; 40: 1041-1049.

${ }^{45} \mathrm{C}$. Morris \& L. Holloway. Genetic technologies and the transformation of the geographies of UK livestock agriculture: a research agenda. Prog Hum Geogr 2009; 33: 313-333.

${ }^{46} \mathrm{R}$. Twine. Animal genomics and ambivalence: a sociology of animal bodies in agricultural Biotechnology. Genomics, Society and Policy 2006; 6: 99-117.

${ }^{47}$ S. MacLean \& M.M. Burgess. In the public interest: Assessing expert and stakeholder influence in public deliberation about biobanks. Pub Understand Sci, in press.

${ }^{48}$ Wynne, B. (2006). Public engagement as a means of restoring public trust in science--hitting the notes, but missing the music? Community Genetics, 9(3), 211-220.

${ }^{49}$ Burgess, O’Doherty \& Secko, op. cit 17.

${ }^{50}$ Ibid; Longstaff \& Burgess, op. cit. 32; O’Doherty \& Burgess, op. cit. 43. 